\title{
PREVALÊNCIA DO USO DE COCAÍNA E MACONHA POR METALÚRGICOS EM CAXIAS DO SUL
}

\author{
PREVALENCE OF COCAINE AND MARIJUANA USE BY METALLURGICAL IN \\ CAXIAS DO SUL
}

Tatiana Thomazzoni Piccoli, Deise Pretto, Adriana Dalpicolli Rodrigues

\section{RESUMO}

Avaliar a prevalência do uso de cocaína e maconha por funcionários metalúrgicos de Caxias do Sul/ RS. Foram analisados resultados de dosagens de cocaína e maconha na urina de 1.223 metalúrgicos em um banco de dados de um laboratório da cidade, em fevereiro e julho de 2014 . Em fevereiro, dos 634 estudados, observou-se positividade de 1,10\% para o uso de cocaína e 2,11\% de maconha. Já em julho, dentre os 589 trabalhadores avaliados, houve positividade de $0,51 \%$ para cocaína e 1,02\% para maconha. Houve predominância de consumo pelo sexo masculino, na faixa etária de 18 a 33 anos (68,80\% em fevereiro e 58,06\% em julho). Embora haja relatos na literatura sobre as possíveis motivações para o uso de drogas ilícitas no setor metalmecânico, como forma de escape diante de esforços físicos constantes ou pressões psicológicas, os resultados encontrados no presente estudo não indicam o uso significativo de cocaína e maconha.

Descritores: Indústria Metalúrgica; Cocaína; Maconha.

\section{ABSTRACT}

Evaluate the prevalence of the use of cocaine and marijuana by metallurgical employees of Caxias do Sul/RS. Analysis of the doses of cocaine and marijuana in the urine of 1.223 metallurgical employees in a databank of a laboratory of the city, in february and july in 2014. In february, 634 people were tested, was observed $1.10 \%$ tested positive for cocaine and $2.11 \%$ for marijuana. In july, among the 589 workers tested, there was positive result in $0.51 \%$ for cocaine and $1.02 \%$ for marijuana. The research observed that most of the people who tested positive were men in the age range 18 to $33(68.80 \%$ in february and $58.06 \%$ in july). Although there are accounts in literature about possible motivations for the use of illegal drugs at metallurgical industry, as a way to escape from constant physical efforts or psychological pressure, the results found in our study do not indicate the significant use of cocaine and marijuana among the workers tested.

Descriptores: Metalmechanic Industry; Cocaine; Maconha.
Tatiana Thomazzoni Piccoli,: Bacharel em Biomedicina pelo Centro Universitário da Serra Gaúcha. Caxias do Sul, Rio Grande do Sul, Brasil.

Deise Pretto, Mestra em Biociências e Reabilitação pelo Centro Universitário Metodista - IPA, Porto Alegre, Rio Grande do Sul, Brasil.

Adriana Dalpicolli Rodrigues, Mestre em Biotecnologia pela Universidade de Caxias do Sul. 


\section{Introdução}

O Brasil é considerado uma das principais rotas de distribuição de drogas ilícitas do Continente Americano. 0 fato de fazer fronteira com os principais países produtores destas substâncias e a precariedade da fiscalização nas divisas e o que torna viável a instalação do mercado de drogas no país. ${ }^{1}$

O município de Caxias do Sul está localizado na região Nordeste do Rio Grande do Sul e conta com aproximadamente 435.564 mil habitantes, conforme o último Censo de $2010 .{ }^{2} \mathrm{~A}$ cidade tem o segundo maior polo industrial do Brasil, o qual corresponde a $42,55 \%$ da renda municipal. No total, são 3.779 empresas registradas e 71.972 postos de trabalho de janeiro a dezembro do ano de 2014, nos setores automotivo, eletroeletrônico e metalomecânico ${ }^{3,4}$

Araújo e Oliveira ${ }^{5}$ apontam que muitas empresas do setor metalmecânico passam por um processo de reestruturação produtiva. Isso inclui uma inovação tecnológica dentro do chão de fábrica, com equipamentos modernos, processos automatizados e novos métodos gerenciais. Todavia, o ritmo de trabalho intenso e a pressão sofrida sobre os metalúrgicos ainda continuam, aliados a um maior comprometimento com as metas de produção. Diante deste cenário o uso de substâncias ilícitas dentro do setor industrial, como o metalmecânico, torna-se cada vez mais contundente, servindo como um meio de escape diante das atuais condições de trabalho, sendo a maconha e a cocaína as mais consumidas. ${ }^{1}$

Almeida et a $\left.\right|^{6}$ mostraram que dependentes de maconha têm comprometimentos cognitivos parecidos aos encontrados em pacientes com lesões frontais do cérebro. A dificuldade de mudar de uma metodologia de trabalho para outra, a incapacidade de absorver detalhes isolados e de usar informações ao mesmo tempo, fazem com que o rendimento dessa pessoa no ambiente de trabalho fique prejudicado. No indivíduo, as funções executivas são determinadas como um conjunto de ações nas quais estão processos cognitivos, emocionais, motivacionais e volitivos. ${ }^{6}$

Segundo Lima ${ }^{7}$ existem dois casos para explicar o uso de drogas: para relaxar após uma jornada de trabalho ou para sentir a sensação de euforia. Esta segunda opção ao uso de drogas reflete os tipos de trabalho exercidos pelo funcionário. Por serem cada vez mais duros, geram um aumento de estresse e desinteresse, principalmente diante da ausência de reconhecimento. Contudo, o uso crônico dessas substâncias pode se tornar disfuncional no contexto do trabalho e gerar punições, transferências, rebaixamento de função, imposição de tarefas menos interessantes ou isolamento pelo próprio funcionário.

A Norma Regulamentadora - NR7 do Programa de Controle Médico de Saúde Ocupacional (PCMSO) impõe ao trabalhador realizar exames periódicos, sendo eles: admissional, periódico, retorno ao trabalho, mudança de função demissional.8 Em vista disso, desde 1992, empresas passaram a solicitar exames de drogas na urina dos funcionários. Embora não sejam obrigatórios, houve uma grande preocupação do empregador em relação ao uso de drogas no ambiente de trabalho por afetar o comportamento e a saúde do trabalhador.1 Portanto, o presente estudo teve como objetivo avaliar a prevalência do uso de cocaína e maconha em funcionários metalúrgicos da cidade de Caxias do Sul/ Rio Grande do Sul, Brasil.

\section{Metodologia}

Foi realizado um estudo descritivo transversal retrospectivo, tendo como público alvo trabalhadores de indústrias metalúrgicas da cidade de Caxias do Sul/Rio Grande do Sul, Brasil.

Foram incluídos no estudo os resultados de detecção de cocaína e maconha na urina de trabalhadores de ambos os sexos, com faixa etária de 18 a 65 anos, que partiram de um banco de dados de um laboratório de análises clínicas da mesma cidade, correspondentes aos meses de fevereiro e julho de 2014. Estes dois meses foram selecionados para possibilitar a comparação de resultados entre um mês de cada semestre, sendo um de verão, em que ocorre um maior número de festividades e outras atividades de entretenimento nas quais os funcionários poderiam sentir maior necessidade do uso de droga, com um mês de inverno, relativamente com menos atividades. Além disso, foram os meses em que o número de amostras analisadas no laboratório foi muito próximo (Fevereiro $n=634$; Julho $n=589$ ), facilitando a comparação e diferenciando-se dos outros meses do ano. Foram excluídos do estudo menores de 18 anos e profissionais recém contratados, temporários ou estagiários. Para análise estatística foi utilizado o teste qui-quadrado $(\mathrm{p}<0,05)$, no programa SPSS 20.0 .
O laboratório de análises clínicas que forneceu o banco de dados faz a análise destas positividades com o método de teste rápido imunocromatográfico de fluxo lateral, baseado no princípio de vínculos competitivos. Para a detecção qualitativa de drogas de abuso e seus metabólitos, as concentrações são de $50 \mathrm{ng} / \mathrm{ml}$ para maconha e $150 \mathrm{ng} / \mathrm{ml}$ para cocaína.9 É importante ressaltar que todas as amostras dos testes que resultaram positividade nesta técnica, foram encaminhadas a um outro laboratório de apoio de referência nacional, para realização de um método químico mais específico a fim de obter um resultado analítico confirmatório.

O presente estudo foi aprovado pelo Comitê de Ética em Pesquisa (CEP) do Círculo/FSG, segundo as Normas de Pesquisa Envolvendo Seres Humanos (Resolução 466/12) do Conselho Nacional de Saúde, sob o número 894.572.

RESULTADOS:

A tabela 1 descreve os resultados do uso de cocaína e maconha pelos funcionários nos meses de fevereiro e julho (2014), com uma amostra total de 1.223 indivíduos. No mês de fevereiro, foram avaliadas 634 amostras, sendo que o predomínio foi do sexo masculino $(91,17 \%)$, e a média de idade ficou em $30,76 \pm 8,94$ anos. 0 uso de cocaína ficou evidenciado em $1,10 \%$ da amostra total, sendo que para maconha observou-se uma quantidade um pouco maior $(2,21 \%)$, o que representa uma diferença de $1,11 \%$ entre as duas drogas. Dos 634 indivíduos, dois do sexo masculino mostraram testes positivos tanto para cocaína quanto para maconha $(0,31 \%)$.

Tabela 1 Perfil de trabalhadores metalúrgicos nos meses de Fevereiro $(n=634)$ e Julho (n=589). Caxias do Sul, Rio Grande do Sul, 2014

\begin{tabular}{lccccccc}
\hline \multirow{2}{*}{ Variáveis } & \multirow{2}{*}{ Categoria } & \multicolumn{3}{c}{ Fevereiro } & \multicolumn{2}{c}{ Julho } & \multicolumn{2}{c}{ Total } \\
\cline { 3 - 8 } & $n(634)$ & $\%$ & $n(589)$ & $\%$ & $n$ & $(1.223)$ & $\%$ \\
\hline Sexo & Feminino & 56 & 8,83 & 36 & 6,11 & 92 & 7,52 \\
& Masculino & 578 & 91,17 & 553 & 93,89 & 1.131 & 92,48 \\
Fixa etária & & & & & & & \\
& $18-33$ & 436 & 68,80 & 342 & 58,06 & 778 & 63,62 \\
& $34-49$ & 178 & 28,05 & 207 & 35,14 & 385 & 31,48 \\
& $50-65$ & 20 & 3,15 & 40 & 6,80 & 60 & 4,90 \\
Cocaína & & & & & & & \\
& Positivo & 7 & 1,10 & 3 & 0,51 & 10 & 0,81 \\
& Negativo & 627 & 98,90 & 586 & 99,49 & 1.213 & 99,19 \\
Maconha & Positivo & 14 & 2,21 & 6 & 1,02 & 20 & 1,63 \\
& Negativo & 620 & 97,79 & 583 & 98,98 & 1.203 & 98,37 \\
\hline
\end{tabular}

O mês de julho teve um número amostral de 589 análises e, igualmente ao mês de fevereiro, foi predominantemente masculino (93,89\%). O uso de drogas de abuso teve resultado menor, para cocaína um percentual de $0,51 \%$ e maconha de $1,02 \%$. Neste mês, a média de idade foi de $32,50 \pm 10,19$ anos. Vale ressaltar que dentre toda a população apresentada, somente a amostra de um indivíduo resultou positiva para as duas drogas de abuso $(0,16 \%)$, sendo este do sexo masculino.

A faixa etária que mais fez uso tanto de maconha quanto de cocaína foi dos 18 aos 33 anos (81,48\%), em fevereiro e julho, representado na tabela 2. A positividade dessas drogas no sexo feminino também foi detectada. Dentre 56 dosagens na urina das mulheres no mês de fevereiro, uma apresentou resultado positivo para o uso de maconha $(1,79 \%)$ (tabela 3). 
Tabela 2 Positividade de cocaína e maconha por faixa etária entre trabalhadores metalúrgicos nos meses de Fevereiro $(n=20)$ e Julho $(n=10)$. Caxias do Sul, Rio Grande do Sul, 2014

\begin{tabular}{cccc} 
Variável & Categoria & $n(30)$ & $\%$ \\
\hline Fixa etária & $18-33$ & 24 & 81,48 \\
& $34-49$ & 6 & 18,52 \\
& $50-65$ & 0 & 0
\end{tabular}

Tabela 3 Positividade de cocaína e maconha por sexo entre trabalhadores metalúrgicos nos meses de Fevereiro $(n=634)$ e Julho $(n=589)$. Caxias do Sul, Rio Grande do Sul, 2014

\begin{tabular}{|c|c|c|c|c|c|c|c|c|c|c|c|c|c|}
\hline \multirow{3}{*}{ Variáveis } & \multirow{3}{*}{ Categoria } & \multicolumn{4}{|c|}{$\begin{array}{c}\text { Fevereiro } \\
\text { Feminino }(n=56) \\
\text { Masculino }(n=578)\end{array}$} & \multicolumn{4}{|c|}{$\begin{array}{c}\text { Julho } \\
\text { Feminino }(n=36) \\
\text { Masculino }(n=553)\end{array}$} & \multicolumn{4}{|c|}{$\begin{array}{c}\text { Total } \\
\text { Feminino }(n=92) \\
\text { Masculino }(n=1.131)\end{array}$} \\
\hline & & \multicolumn{2}{|c|}{ maconha } & \multicolumn{2}{|c|}{ cocaína } & \multicolumn{2}{|c|}{ maconha } & \multicolumn{2}{|c|}{ cocaína } & \multicolumn{2}{|c|}{ maconha } & \multicolumn{2}{|c|}{ cocaína } \\
\hline & & $\begin{array}{c}n \\
(14)\end{array}$ & $\%$ & $\begin{array}{c}n \\
(7)\end{array}$ & $\%$ & $\begin{array}{c}n \\
(6)\end{array}$ & $\%$ & $\begin{array}{c}n \\
\text { (3) }\end{array}$ & $\%$ & $\begin{array}{c}n \\
(20)\end{array}$ & $\%$ & $\begin{array}{c}n \\
(10)\end{array}$ & $\%$ \\
\hline \multirow[t]{2}{*}{ Sexo } & Feminino & 1 & 1,79 & 0 & 0 & 0 & 0 & 0 & 0 & 1 & 1,08 & 0 & 0 \\
\hline & Masculino & 13 & 2,25 & 7 & 1,21 & 6 & 1,08 & 3 & 0,54 & 19 & 1,68 & 10 & 0,88 \\
\hline
\end{tabular}

Foi realizada uma comparação entre os meses de fevereiro e julho, porém os resultados não tiveram significância estatística (dados não mostrados).

Discussão

O presente estudo mostrou uma prevalência relativamente baixa do uso de cocaína e maconha. Foram encontradas positividades em 30 trabalhadores, o que correspondeu a 2,44\% dentre os 1.223 metalúrgicos avaliados, da cidade de Caxias do Sul/RS no ano de 2014, nos meses de fevereiro e julho. DuPont et. al.,10 em seu estudo, concluiu que, por ano, a taxa estimada de positividade de drogas ilícitas na força de trabalho é de quase oito vezes a taxa de testes aleatórios também positivos. Se durante um ano uma empresa apresenta $1 \%$ de positividade em testes aleatórios para drogas, pode supor que $8 \%$ de todo seu âmbito empregatício usa drogas pelo menos uma vez no mesmo ano, o que corrobora com a quantidade de consumo dos achados desse estudo.

No âmbito mundial, a maconha se enquadra como a droga ilícita mais consumida. Na América do Sul, chega a $3 \%$ na faixa etária dos 15 a 64 anos em relação ao seu consumo. 11 Nosso estudo está de acordo com esses dois dados, onde mostrou-se que o uso de maconha (2,21\% em fevereiro e $1,02 \%$ em julho) foi maior que o de cocaína $(1,10 \%$ em fevereiro e 0,51\% em julho), com predomínio para a faixa etária dos 18 aos 33 anos, em ambos os meses.

No ambiente de trabalho a nível internacional, um estudo realizado no Reino Unido procurou saber a frequência de drogas utilizadas no trabalho e mostrou que, dentre 1.617 amostras avaliadas, vindas de 82 locais distintos, a maconha foi a mais encontrada (188 amostras/11,6\%) e a cocaína apareceu em 18 amostras (1,1\%), dentre outras drogas que também foram constatadas. 12 Da mesma forma, mas com menor prevalência, nosso estudo relatou um total de 20 amostras positivas para maconha $(1,63 \%)$ e 10 amostras para cocaina $(0,81 \%)$, num total de 1.223 trabalhadores avaliados entre os dois meses citados.

Em um estudo que abordou o consumo de drogas em trabalhadores de diferentes regiões do Brasil, a região Sul foi a que detectou menor índice de uso, sendo que dessas substâncias somente a maconha foi encontrada em cinco amostras $(0,5 \%)$, dentre as 1.003 avaliadas. 1 Diferentemente a estes resultados, o presente estudo, realizado em uma cidade do sul do país, além da maconha, encontrou positividade também no uso de cocaína nos trabalhadores, com
$1,10 \%$ ( 7 amostras) em fevereiro e $0,51 \%$ ( 3 amostras) em julho, haja visto o baixo percentual encontrado tanto para cocaína quanto para maconha, como na pesquisa de Yonamine e Silva. 1

O II Levantamento domiciliar sobre o uso de drogas psicotrópicas no Brasil avaliou 18 cidades do Sul do país, entre elas o município de Caxias do Sul. Dentre os 879 entrevistados, foi constatado um consumo expressivo de maconha em relação a outras drogas de abuso. O sexo masculino foi o que mais utilizou a maconha $(21,6 \%)$ contra o uso pelo sexo feminino (13,3\%), todos na faixa etária dos 18 aos 24 anos. A cocaína também estava presente, com percentual de uso em $9,0 \%$ do sexo masculino e $2,1 \%$ do sexo feminino. 13 No presente estudo também foram encontrados resultados maiores para maconha do que para cocaína e a faixa etária também se concentrou entre os mais jovens. Em relação ao sexo, evidenciou-se prevalência maior em homens, devido a ser o sexo predominante no setor metalúrgico, e também decorrente a ser o sexo com maior perfil ao uso de maconha, igualmente aos achados de Carlini et al.13

Nossa pesquisa revelou um predomínio de indivíduos trabalhadores na faixa etária entre 18 a 33 anos $(68,80 \%$ em fevereiro e $58,06 \%$ em julho), corroborando com os resultados de Dornelles Filho et al. 14 em que as novas contratações no ambiente fabril também eram de jovens de 18 a 24 anos, e que as demissões se concentravam na população com mais idade. Nosso estudo também identificou uma quantidade relativamente menor de trabalhadores dos 50 a 65 anos ( $3,15 \%$ em fevereiro e 6,80\% em julho), sendo a faixa etária em que não se encontrou o uso de cocaína e maconha.

Nos meses de fevereiro e julho observaram-se diferenças nos resultados encontrados, embora não significativas estatisticamente. Houve uma menor prevalência de consumo de drogas em julho $(1,02 \%$ em julho contra $2,21 \%$ em fevereiro). Importante ressaltar que a faixa etária dos 18 aos 33 anos em fevereiro (68,80\%) foi apontada como a que mais fez o uso de substâncias ilícitas no estudo, estando de acordo com a hipótese inicial de que o período do verão, em que há maior número de festividades e outras atividades, também predispõe ao maior uso de drogas.

Tsanaclis, Wicks e Chasin15 explicam que o exame de drogas ilícitas em urina é a técnica mais utilizada e evidencia positividade em indivíduos em um período de 24 a 72 horas após o consumo, dependendo do tipo de droga. Segundo a OBID16 a maconha pode ser encontrada na urina de três dias a quatro semanas, dependendo do uso. Já a cocaína tem seu tempo de detecção de 6 a 8 horas, e seus metabólitos de dois a quatro dias. 0 fato de não termos encontrado diferença estatística, junto com a baixa prevalência do uso de drogas no presente estudo, pode estar relacionada ao período de abstinência dos metalúrgicos no consumo destas drogas, visto que essa é uma informação não contida no banco de dados e nem mesmo solicitada pela empresa, que solicita essas dosagens periodicamente.

Silva e Yonamine1 apontam que as diferenças econômicas, sociais e culturais entre as regiões do Brasil também influenciam no alto ou baixo índice no consumo das drogas ilícitas. Desse modo, são necessários outros estudos que explorem ainda mais as questões que envolvem o uso de drogas ilícitas no ambiente de trabalho metalúrgico, como por exemplo, elaboração de questionários e divisão de trabalhadores por turno de trabalho, haja vista que no setor metalúrgico existem muitos trabalhadores no turno da noite, condições socioeconômicas diferentes e divisões por escolaridade. A ausência dessas informacões pode ser um fator importante relacionado ao baixo índice de consumo de drogas encontrado neste estudo, visto que não foi possível acesso a esses dados.

Considerações Finais

Embora haja relatos na literatura sobre as possíveis motivações para o uso de drogas ilícitas no setor metalmecânico, como forma de escape diante de esforços físicos constantes ou pressões psicológicas, os resultados encontrados não indicaram o uso significativo de cocaína e maconha. Isso pode ocorrer devido ao ambiente de trabalho no município de Caxias do Sul ser considerado sugestivamente benéfico ao funcionário, com uma boa relação de convívio entre estes trabalhadores e as empresas fabris.

Um dos fatores que possibilitam esse resultado é a adoção de políticas públicas pelas empresas, o que deve ser cada vez mais incentivada por meio de estudos como este, que relacionem o uso das drogas de abuso com o trabalho metalúrgico. Por meio do acompanhamento na rotina dos funcionários metalúrgicos, pelos exames ocupacionais, é possível identificar o consumo de drogas e, desta forma, viabilizar o auxílio adequado aos trabalhadores, quando necessário. Por fim, estudos nessa área se mostram de fundamental importância, somando-se aos escassos trabalhos existentes sobre o tema, visando a melhoria da qualidade de vida e de mão de obra dos trabalhadores, fatores também importantes para as empresas empregadoras. 


\section{Referências}

1. Silva O, Yonamine M. Drug abuse among workers in brazilian regions. Rev Saúde Públ. 2004; 38(4):552-556.

2. IBGE - Instituto Brasileiro de Geografia e Estatística. Cidades, 2014. Disponível em: http://www.cidades.ibge. gov.br/xtras/perfil.php?lang=\&codmun=430510.

3. Prefeitura Municipal De Caxias Do Sul. PIB, 2015. Disponível em: <https://www.caxias.rs.gov.br/desenv_ economico/texto.php?codigo=217>.

4. SIMECS - Sindicato das Indústrias Metalúrgicas, Mecânicas e de Material Elétrico de Caxias do Sul. Resultados econômicos, 2014. Disponível em: <http://www.simecs.com.br/empresas/resultados-economicos/>.

5. Araújo A, Oliveira E. Reestruturação produtiva e saúde no setor metalúrgico: a percepção das trabalhadoras. Soc Estado. 2006; 21(1):169-198.

6. $\quad$ Almeida PP, Novaes MAFP, Bressan RA, Lacerda ALT. Revisão: funcionamento executivo e uso de maconha. Rev Bras Psiquiatr. 2008; 30(1):69-76.

7. Lima MEA. Dependência química e trabalho: uso funcional e disfuncional de drogas nos contextos laborais. Rev Bras Saúde Ocup. 2010; 35(122):260-268.

8. Ministério Do Trabalho E Emprego. NR 7 - Programa de Controle Médico de Saúde Ocupacional. Disponível em: <http://portal.mte.gov.br/data/files/FF8080814295F16D0142E2E773847819/NR-07\%20(atualizada\%202013).pdf>.

9. Abon - Abon biopharm Hangzhou Economic \& Technological Development Area. Dispositivo para Teste em MultiLinhas Em Um Só Passo (Urina). Bula de kit; China, 2012.

10. Dupont RL, Griffin DW, Siskin BR, Shiraki S, Katze E. Random drug tests at work: the probability of identifyingfrequent and infrequent users of illicit drugs. J Addict Dis. 1995;14(3)1-17.

11. ONU - Organização das Nações Unidas. Perfil mundial, 2014. Disponível em: <www.onu.org.br>.

12. George S. A snaptshot of workplace drug testing in the UK. Ocup Med. 2005; 1(55):69-71.

13. Carlini EA, Galduróz JCF, Noto AR, Nappo SA. II Levantamento domiciliar sobre o uso de drogas psicotrópicas no Brasil: estudo envolvendo as 108 maiores cidades do país: 2005 - São Paulo: CEBRID - Centro Brasileiro de Informação sobre Drogas Psicotrópicas: UNIFESP - Universidade Federal de São Paulo, 2006.

14. Dornelles Filho A, Dalponte DG, Soares LMPC, Waismann M. Os reflexos financeiros de 2008 no perfil dos novos postos de trabalho criados em Caxias do Sul no período de Setembro de 2008 a Setembro de 2009. Disponível em: $\langle$ ttps://www.ucs.br/site/midia/arquivos/artigo_crise_financeira.pdf $>$.

15. Tsanaclis LM, Wicks JFC, Chasin AAM. Workplace drug testing, different matrices different objectives. Drug Test Anal. 2012; 4(2):83-88.

OBID - Observatório Brasileiro de Informações sobre Drogas. Exames Toxicológicos, 2015. Disponivel em: <http://obid.senad.gov.br/portais/OBID/conteudo/index.php?id_ conteudo $=11252 \&$ rastro $=$ INFORMA $\%$ C3\%87\%C3\%95ES+SOBRE+DROGAS/Exames+toxicol\%C3\%B3gicos $>$. 